\title{
LA PRODUCCION DE LA UNIVERSIDAD ESPAÑOLA EN FISICA, REFLEJADA EN LAS PUBLICACIONES ESPAÑOLAS Y EXTRANJERAS
}

\author{
J. R. Pérez Alvarez-Ossorio; M. I. Gómez Caridad; M. J. Martín Sempere; \\ C. Galbán Ferrús; M. C. Urdin Caminos y A. I. Sobrado Presa*
}

Resumen: Se analiza la producción científica de la Universidad española en el campo de la Física, y en el período 1986-88, a través de dos bases de datos: INSPEC, que recoge lo publicado en revistas extranjeras y también, aunque de modo selectivo, en cierto número de revistas españolas; e ICYT, que recoge exhaustivamente todos los trabajos originales publicados en revistas españolas. La comparación entre ambas series de datos permite deducir tendencias de publicación en el extranjero. Asimismo se analizan comparativamente los periodos 1982-85 y 1986-88, con los datos obtenidos de INSPEC.

Palabras clave: Física, producción científica, publicaciones cientificas, Universidad, España.

\begin{abstract}
The scientific production of Spanish Universities in the field of Physics for the period 1986-88 is analyzed with data obtained from two databases: INSPEC, which covers publications in foreign journals and also, but only in a selective way, those published in a certain number of Spanish journals; and ICYT, which covers comprehensively all original papers published in Spanish journals. Comparison of the two series of data leads to certain conclusions as regards the trends to publish in foreign journals. A further comparison is made between the periods $1982-85$ and 1986-88, with the data obtained from INSPEC.
\end{abstract}

Keywords: Physics, scientific out-put, scientific publications, University, Spain.

\section{Introducción}

El objetivo de este trabajo consiste en analizar la producción de la Universidad española en el área de la Física y en el período 1986-88, a través de los trabajos publicados en la literatura científico-técnica, tanto española como extranjera. Se han escogido para ello dos bases de datos que se consideran respectivamente representativas para uno y otro casos: la base de datos INSPEC, la más conocida y exhaustiva de las bases de datos internacionales en el campo de la Física, donde se recogen los trabajos españoles que aparecen en publicaciones extranjeras y también (aunque no de modo exhaustivo) en cierto número de revistas españolas y que representa, de algún modo, un índice de la producción española que alcanza difusión internacional. Y la base de datos ICYT, donde se recogen exhaustivamente los trabajos publicados en revistas españolas.

El trabajo consta, pues, de tres partes:

1. Estudio de las aportaciones de la Universidad española recogidas por INSPEC.

* Instituto de Información y Documentación en ('iencia y Tecnologia (ICYT). Madrid (CSIC).

Recibido 13-5-91. 
2. Análisis de los trabajos de Física, procedentes de la Universidad, que se recogen en la base de datos ICYT.

3. Comparación de los resultados de las dos primeras partes, que permitirá deducir las proporciones relativas de la producción de la Universidad española en Física que se publica en revistas españolas y extranjeras.

\section{Análisis de los datos procedentes de la base de datos INSPEC}

\section{Metodologia}

La base de datos INSPEC, creada en el Reino Unido por la Institution of Electrical Engineers, cubre publicaciones de física, ingenieria eléctrica y electrónica y ordenadores procedentes de 4.100 revistas científicas de todo el mundo.

Se recuperaron las referencias de aquellos documentos en que figura España como lugar de trabajo, cuyo año de publicación corresponde a 1986, 1987 y 1988. Se teledescargaron los documentos y se crearon tres bases de datos, una por cada año analizado, en KNOSYS. Dada la falta de homogeneidad en los nombres de las instituciones, éstas se codificaron manualmente, para identificar facultades, escuelas y otras dependencias de la Universidad.

Hay que tener en cuenta que INSPEC recoge sólo la institución correspondiente al primer firmante de cada trabajo, lo que conduce a los siguientes problemas:

a) Se pierden aquellos trabajos realizados en colaboración entre centros españoles y extranjeros, cuando el autor español no figura en primer lugar. Sin embargo, como la inmensa mayoría de estos casos corresponde a trabajos realizados fuera de España, que no se contemplan aquí, el error eventual debido a esta causa sería mínimo.

b) Los trabajos realizados en colaboración por varias instituciones españolas se asignan siempre a la institución del primer autor. Según un muestreo realizado, cuando un centro universitario colabora con otro extra-universitario, en la gran mayoria de los casos aquél figura en primer lugar. Por ello, la posible pérdida de trabajos es mínima y, por otra parte, como lo que aquí se estudia es la aportación global de la Universidad, es correcto computar estos trabajos. Pero esta circunstancia sí afecta al porcentaje que corresponde a la Universidad frente a otras instituciones españolas, que resultan perjudicadas en este cómputo. Por ello, cabe únicamente hablar del porcentaje del total de trabajos españoles en que ha participado la Universidad.

c) Cuando se trata de colaboraciones entre varios centros universitarios, los trabajos se asignan igualmente al centro del primer firmante. Por tanto, todas las distribuciones están hechas en función de estos centros "primeros firmantes", circunstancia que hay que tener en cuenta para la correcta interpretación de los resultados.

d) Se asignaron a la Universidad, en el presente estudio, los trabajos procedentes de centros mixtos, como puede ser el Instituto de Astrofisica de Canarias o Centros de Materiales. 


\section{Resultados}

Se obtuvieron de INSPEC un total de 5.157 referencias de documentos españoles, incluyendo una gran mayoría de artículos de revista (3.646), comunicaciones presentadas a congresos (1.508) y solamente 3 libros (Fig. 1). La Universidad participó en 4.213 de estos documentos, distribuidos muy desigualmente entre facultades y escuelas técnicas: los artículos de revista proceden en un $82 \%$ de las facultades, mientras que la distribución de los congresos entre facultades y escuelas está mucho más igualada (Fig. 2).

Teniendo en cuenta que las comunicaciones a congresos son resultados preliminares de investigación que suelen dar lugar a la larga a artículos publicados en revistas y que, por otra parte, la base de datos ICYT incluye sólo artículos de revista, limitaremos nuestro análisis a este último tipo de documentos.

La figura 3 recoge la distribución global de los artículos de revista procedentes de España, diferenciando aquellos en los que ha participado la Universidad. A lo largo del trienio esta participación se mantiene bastante estable: varía del 85 al $82 \%$ de la producción total.

Figura 1

Documentos espanoles en INSPEC en el trienio 1986-88

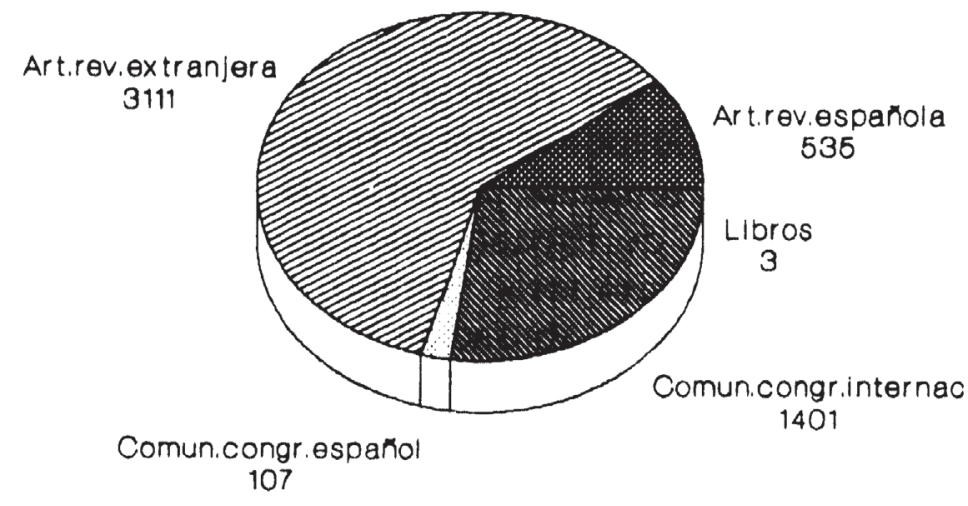

Figura 2

Documentos de la Universidad en INSPEC en el trienio 1986-88
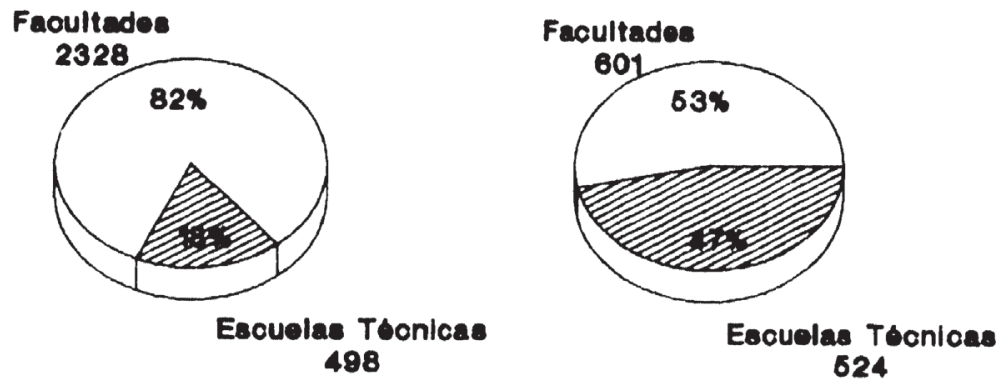

Artículos de revista

Comunicaciones a congresos 
Figura 3

Participación relativa de la Universidad en INSPEC (articulos de revista)

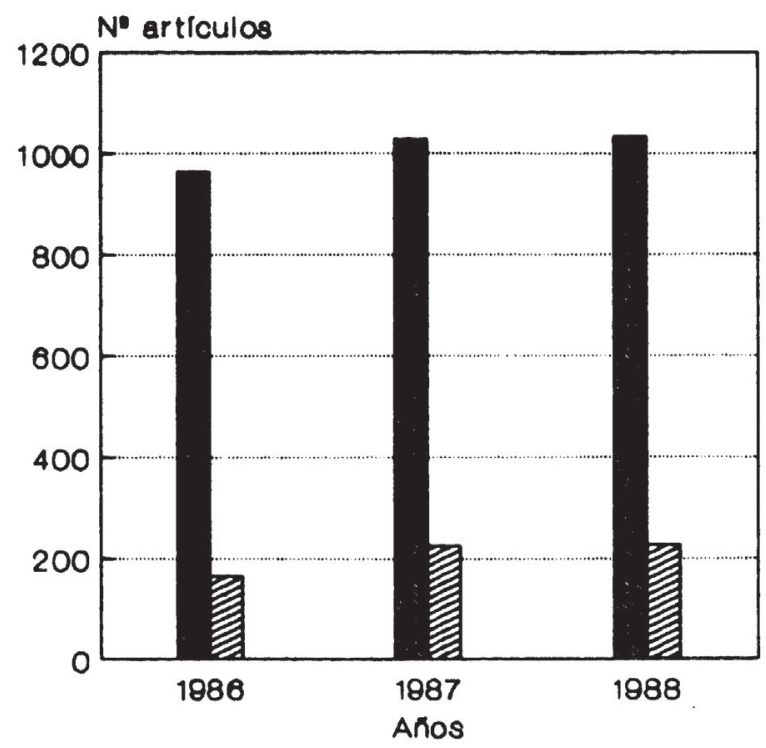

Univer sldad

No Universidad

A continuación se ha procedido a distribuir los trabajos por tipos de centros y por Universidades (tablas I y II).

En la tabla I se presenta la producción anual de las facultades y escuelas técnicas superiores (ETS). Dentro de un pequeño aumento general a lo largo del período (en torno al $7 \%$ ) éste es mayor para las ETS, incluida Informática (en torno al $10 \%$ ) que para las facultades (en torno al $5,5 \%$ ).

Con objeto de cuantificar la contribución individual de los centros de los distintos tipos, se han incluido, en las dos últimas columnas de la tabla I, el número de centros productores de cada tipo y el promedio de artículos por centro. Según este último criterio, figuran destacadamente a la cabeza las facultades de Física y las escuelas de Ingenieros de Telecomunicación. Siguen a considerable distancia las facultades de Química, las escuelas de Ingenieros Aeronáuticos y las Escuelas de Ingenieros Industriales; aunque hay que tener en cuenta que en el caso de los Ingenieros Aeronáuticos se trata de una sola Escuela. Algo similar ocurre en el caso de los Institutos Universitarios, ya que, de los 72 trabajos, 62 corresponden a un solo centro, el Instituto de Astrofísica de Canarias.

La tabla II recoge la distribución de los artículos de revista por Universidades. Destacan como más productivas la Universidad Complutense de Madrid, seguida de la Autónoma de Madrid y la Central de Barcelona.

\section{Comparación con períodos anteriores}

En los últimos años se ha publicado una serie de trabajos sobre la producción científica española en el área de la Física, tanto partiendo de la información de la base de datos INSPEC $(1,2,3)$, como utilizando la base de datos Physics Brief (4), 
Tabla I

Artículos de revista por tipo de centro y ano, y promedios por centro (INSPEC)

\begin{tabular}{lrrrrrr}
\hline & 1986 & 1987 & 1988 & Total & $\begin{array}{c}\text { Núm. de } \\
\text { centros }\end{array}$ & $\begin{array}{c}\text { Promedio/ } \\
\text { centro }\end{array}$ \\
\hline Fac. de Física & 491 & 530 & 530 & 1.551 & 21 & 74 \\
Fac. de Química & 183 & 183 & 180 & 546 & 25 & 22 \\
Fac. de Matemáticas & 32 & 40 & 40 & 112 & 14 & 8 \\
Fac. de Informática & 15 & 22 & 16 & 53 & 6 & 9 \\
Fac. de Geología & 11 & 15 & 10 & 36 & 9 & 4 \\
Fac. de Medicina & 10 & 7 & 10 & 27 & 8 & 3 \\
Fac. de Biología & 9 & 7 & 5 & 21 & 4 & 5 \\
Fac. de Farmacia & 2 & 6 & 3 & 11 & 4 & 3 \\
Fac. de Veterinaria & 5 & 3 & 2 & 10 & 3 & 3 \\
Otras facultades & 3 & 4 & 7 & 14 & 8 & 2 \\
ETSI Industriales & 63 & 62 & 78 & 203 & 13 & 16 \\
ETSI Telecomunicación & 55 & 42 & 42 & 139 & 2 & 69 \\
ETSI Arquitectura & 10 & 9 & 10 & 29 & 4 & 7 \\
ETSI Caminos & 5 & 9 & 14 & 28 & 3 & 9 \\
ETSI Minas & 3 & 11 & 6 & 20 & 2 & 10 \\
ETSI Aeronáuticos & 6 & 6 & 6 & 18 & 1 & 18 \\
ETSI Agrónomos & 3 & 1 & 4 & 8 & 2 & 4 \\
Institutos Universitarios & 18 & 22 & 32 & 72 & 6 & 12 \\
Esc. Universit. Técnicas & 5 & 19 & 14 & 38 & 10 & 4 \\
Colegios Universitarios & - & 3 & 4 & 7 & 3 & 2 \\
Esc. Universit. no Técnicas & 2 & 3 & - & 5 & 4 & 1 \\
$\quad$ Total & 931 & 1.004 & 1.013 & 2.948 & 152 & 19 \\
Sin especificar & 34 & 24 & 22 & 80 & -5 & - \\
\hline Total general & 965 & 1.028 & 1.035 & 3.028 & & \\
$\quad$ Total Facultades & & & & & & \\
$\quad$ (excl. Informática) & 746 & 795 & 787 & 2.328 & 96 & 24 \\
$\quad$ Total ETS & & & & & & \\
(incl. Informática) & 160 & 162 & 176 & 498 & 33 & 15 \\
\hline & & & & & & \\
\hline
\end{tabular}

o citas recibidas a través del Science Citation Index (5). No todas las series son comparables. Unicamente cabe decir que, si en los datos de 1971-77 la Universidad representa el $68 \%$ del total para España, en 1986-88 ha pasado al $83 \%$. Y que, si en el conjunto de la Universidad, las Escuelas Técnicas representaban sólo el $7,6 \%$ del total, en 1986-88 este porcentaje se ha duplicado (16,3\%).

Los datos correspondientes a los períodos $82-85$ y $86-88$ sí son prácticamente comparables. Sólo hay que tener en cuenta que en el primer período se hizo un recuento fraccionado de los documentos procedentes de centros mixtos CSICUniversidad, adscribiendo en estos casos 0,5 del documento a la Universidad, mientras que en el segundo periodo siempre que participase la Universidad, aun en centros mixtos, se le computó el documento completo. 
Tabla II

Distribución de los artículos de revista por Universidades y anos (INSPEC)

\begin{tabular}{|c|c|c|c|c|}
\hline & 1986 & 1987 & 1988 & Total \\
\hline Complutense & 116 & 98 & 104 & 318 \\
\hline Autónoma Madrid & 85 & 112 & 96 & 293 \\
\hline Barcelona & 85 & 96 & 97 & 278 \\
\hline Zaragoza & 68 & 48 & 66 & 182 \\
\hline Politécnica Cataluña & 68 & 48 & 64 & 180 \\
\hline Politécnica Madrid & 56 & 58 & 61 & 175 \\
\hline Valencia & 44 & 62 & 63 & 169 \\
\hline Sevilla & 43 & 65 & 48 & 156 \\
\hline Pais Vasco & 43 & 52 & 58 & 153 \\
\hline Autónoma Barcelona & 44 & 47 & 48 & 139 \\
\hline Valladolid & 46 & 43 & 35 & 124 \\
\hline Cantabria & 33 & 39 & 26 & 98 \\
\hline La Laguna & 22 & 23 & 40 & 85 \\
\hline Granada & 24 & 30 & 26 & 80 \\
\hline Santiago de Compostela & 25 & 24 & 30 & 79 \\
\hline Oviedo & 27 & 31 & 19 & 77 \\
\hline Salamanca & 25 & 22 & 19 & 66 \\
\hline Politécnica de Valencia & 13 & 21 & 21 & 55 \\
\hline Extremadura & 14 & 14 & 13 & 41 \\
\hline Cádiz & 13 & 17 & 11 & 41 \\
\hline Baleares & 6 & 12 & 21 & 39 \\
\hline Murcia & 12 & 13 & 10 & 35 \\
\hline Córdoba & 7 & 8 & 16 & 31 \\
\hline Alicante & 13 & 7 & 10 & 30 \\
\hline UNED & 8 & 7 & 15 & 30 \\
\hline Castilla-La Mancha & 7 & 10 & 5 & 22 \\
\hline Málaga & 3 & 11 & 8 & 22 \\
\hline Alcalá de Henares & 3 & 3 & 1 & 7 \\
\hline León & 4 & 1 & 2 & 7 \\
\hline Navarra & 3 & 2 & 1 & 6 \\
\hline Deusto & & 4 & $\ldots$ & 4 \\
\hline Politécnica Canarias & 3 & $\ldots$ & 1 & 4 \\
\hline Pontificia Comillas & 1 & $\ldots$ & $-\cdots$ & 1 \\
\hline Internacional Menéndez Pelayo & 1 & $\ldots$ & $\cdots-$ & 1 \\
\hline Total & 965 & 1.028 & 1.035 & 3.028 \\
\hline
\end{tabular}

En la figura 4 se presenta la evolución del número de artículos de la Universidad de 1982 a 1988. Se observa un crecimiento considerable mantenido en todo el período, tanto de artículos procedentes del total de facultades como de escuelas técnicas.

En las tablas III y IV se desglosa la producción de los dos periodos por tipo de centro y por Universidades. En cuanto a la tabla III, hay que advertir que, en las 
Figura 4

Artículos de revista de la Universidad en INSPEC

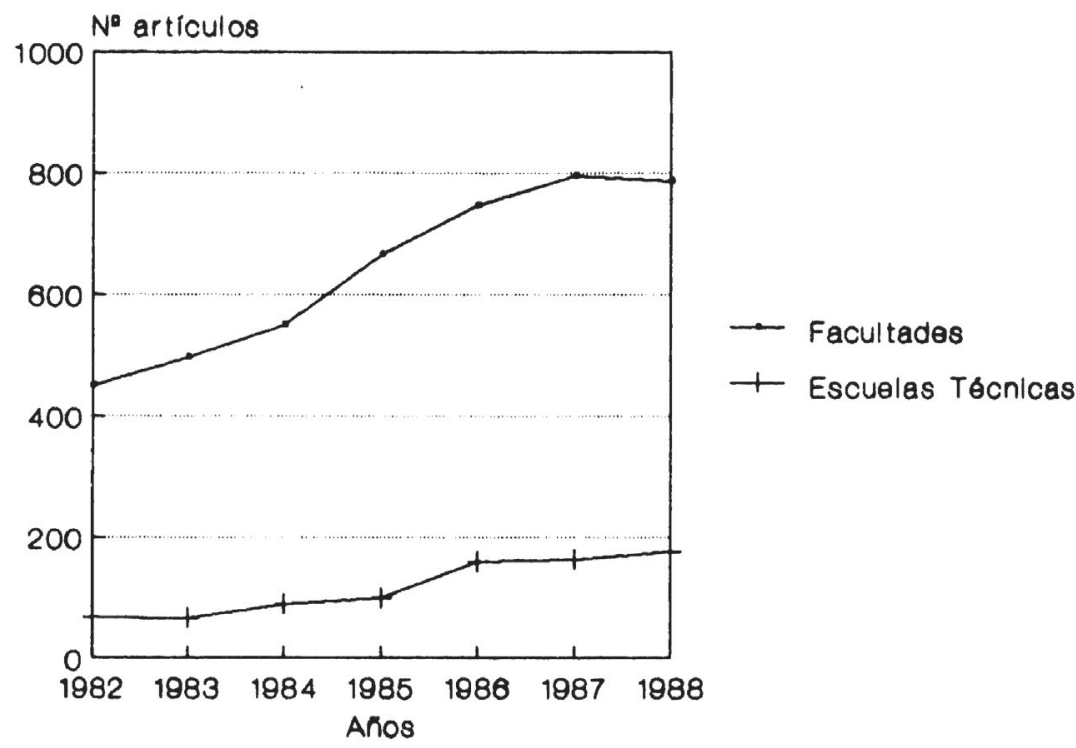

cifras de 1982-85, los datos de los Colegios y Escuelas universitarios aparecen englobados en las especialidades respectivas de facultades y ETS. No obstante, como las cifras correspondientes a aquellos centros son muy pequeñas, el error en que se incurre es despreciable y la comparación referida a facultades y ETS es del todo válida. Como puede verse, el orden se mantiene muy aproximadamente, pudiéndose destacar sólo la subida de las escuelas de Arquitectura y la bajada de la de Aeronáutica. En cuanto a los cuatro tipos principales de centros, el aumento relativo en los promedios por año es notablemente menor en los centros típicamente fisicos (facultades de Física y escuelas de Telecomunicación) que en los que podríamos llamar «afines» (facultades de Química y escuelas de Industriales). Y resalta, particularmente, la gran subida de las ETSI Industriales.

En la tabla IV, las Universidades figuran en orden decreciente según su producción en el período 1986-88. Como dicho período consta de tres años y el anterior de cuatro, se han incluido también los correspondientes promedios por año para ambos períodos. Resalta en primer término la subida de la Universidad de La Laguna, que se debe a la influencia del Instituto de Astrofisica de Canarias, no computado como de la Universidad en el período 82-85. Por lo demás cabe destacar la mayor subida relativa de las Universidades de Cádiz y Politécnica de Cataluña (que pasan de los puestos 28 y 12 a los 20 y 5, respectivamente) y los mayores descensos relativos de las Universidades Autónoma de Barcelona, Alicante y UNED.

\section{Análisis de los datos procedentes de la base de datos ICYT}

\section{Metodología}

La base de datos ICYT, creada en 1979 por el Instituto de Información y Documentación en Ciencia y Tecnología del CSIC, recoge las referencias biblio- 
Tabla III

Artículos en revistas espanolas y extranjeras en los períodos 1982-85 y 1986-88 por tipos de centro (INSPEC)

\begin{tabular}{|c|c|c|c|c|}
\hline & $1982-85^{*}$ & $1986-88^{*}$ & $\begin{array}{c}\text { Promedio/año } \\
1982-85\end{array}$ & $\begin{array}{c}\text { Promedio/año } \\
1986-88\end{array}$ \\
\hline Facultades de Física & $1.602 \quad(1)$ & 1.551 & 400 & 517 \\
\hline Facultades de Química & $420 \quad(2)$ & $546 \quad(2)$ & 105 & 182 \\
\hline Facultades de Matemáticas & $73 \quad(5)$ & $112 \quad(5)$ & 18 & 37 \\
\hline Facultades de Informática & $33 \quad(6)$ & $53 \quad(6)$ & 8 & 18 \\
\hline Facultades de Geología & $25 \quad(7)$ & 36 (7) & 6 & 12 \\
\hline Facultades de Medicina & $12(11)$ & $27(10)$ & 3 & 9 \\
\hline Facultades de Biología & $13(10)$ & $21(11)$ & 3 & 7 \\
\hline Facultades de Farmacia & $6(13)$ & $11(15)$ & 1 & 4 \\
\hline Facultades de Veterinaria & $1(17)$ & $10(16)$ & 0,25 & 3 \\
\hline Otras Facultades & $4(15)$ & $14(14)$ & 1 & 5 \\
\hline ETSI Industriales & 104 (4) & $203 \quad(3)$ & 26 & 68 \\
\hline ETSI Telecomunicación & $127 \quad(3)$ & 139 (4) & 32 & 46 \\
\hline ETSI Arquitectura & $5(14)$ & $29 \quad(8)$ & 1 & 10 \\
\hline ETSI Caminos & $21 \quad(8)$ & $28 \quad(9)$ & 5 & 9 \\
\hline ETSI Minas & $10(12)$ & $20(12)$ & 2 & 7 \\
\hline ETSI Aeronáuticos & $21 \quad(9)$ & $18(13)$ & 5 & 6 \\
\hline ETSI Agrónomos & $3(16)$ & $8(17)$ & 1 & 3 \\
\hline ETSI Navales & $1(18)$ & $-\quad-$ & 0,25 & $\ldots$ \\
\hline $\begin{array}{l}\text { Total Facultades } \\
\text { (excl. Informática) } \\
\text { Total ETS }\end{array}$ & 2.156 & 2.328 & 539 & 776 \\
\hline (incl. Informática) & 325 & 498 & 81 & 166 \\
\hline Total general & 2.481 & 2.826 & 620 & 942 \\
\hline
\end{tabular}

* Entre paréntesis los números de orden que corresponden a cada periodo.

gráficas de los trabajos originales aparecidos en 450 publicaciones científicas españolas, en todos los campos de la Ciencia y la Tecnología. Para el presente estudio se han recogido todas aquellas referencias clasificadas según la Nomenclatura Internacional de UNESCO en los siguientes campos científicos: Física, Astronomía y Astrofisica y, dentro de los campos de Matemáticas y de Ciencias Tecnológicas, las disciplinas de: Ciencia y Tecnología de los Ordenadores, Tecnología Eléctrica, Electrónica, Instrumentación, Nuclear, Energética, del Espacio y de las Telecomunicaciones. Estas temáticas han sido seleccionadas porque son las recogidas en la base de datos INSPEC, con lo que los resultados obtenidos pueden ser comparables.

Se han recuperado las referencias bibliográficas de los artículos publicados por las facultades, colegios universitarios, escuelas técnicas y demás centros universitarios existentes en España. Para cada documento se han recogido todas las instituciones participantes (a diferencia de INSPEC que sólo recoge la primera 
Tabla IV

Comparación entre los períodos $1982-85$ y $1986-88$ por Universidades (INSPEC)

\begin{tabular}{|c|c|c|c|c|}
\hline & $1982-85^{*}$ & $1986-88$ & $\begin{array}{c}\text { Promedio/año } \\
1982-85\end{array}$ & $\begin{array}{c}\text { Promedio/año } \\
1986-88\end{array}$ \\
\hline 1. Complutense & $366 \quad(1)$ & 318 & 91 & 106 \\
\hline 2. Autónoma Madrid & $308 \quad(2)$ & 293 & 77 & 95 \\
\hline 3. Barcelona & $229 \quad(3)$ & 278 & 57 & 93 \\
\hline 4. Zaragoza & 147 (5) & 182 & 37 & 61 \\
\hline 5. Politécnica Cataluña & $99(12)$ & 180 & 25 & 60 \\
\hline 6. Politécnica Madrid & $139 \quad(7)$ & 175 & 35 & 58 \\
\hline 7. Valencia & $111(10)$ & 169 & 28 & 56 \\
\hline 8. Sevilla & $115 \quad$ (9) & 156 & 29 & 52 \\
\hline 9. País Vasco & $140 \quad(6)$ & 153 & 35 & 51 \\
\hline 10. Autónoma Barcelona & $177 \quad(4)$ & 139 & 44 & 46 \\
\hline 11. Valladolid & $124 \quad(8)$ & 124 & 31 & 41 \\
\hline 12. Cantabria & $102(11)$ & 98 & 25 & 33 \\
\hline 13. La Laguna & $10(27)$ & 85 & 2 & 28 \\
\hline 14. Granada & $82(13)$ & 80 & 20 & 27 \\
\hline 15. Santiago de Compostela & $69(14)$ & 79 & 17 & 26 \\
\hline 16. Oviedo & $52(15)$ & 77 & 13 & 26 \\
\hline 17. Salamanca & $38(16)$ & 66 & 9 & 22 \\
\hline 18. Politécnica Valencia & $19(19)$ & 55 & 5 & 18 \\
\hline 19. Extremadura & $19(20)$ & 41 & 5 & 14 \\
\hline 20. Cádiz & $7(28)$ & 41 & 2 & 14 \\
\hline 21. Baleares & $14(23)$ & 39 & 3 & 13 \\
\hline 22. Murcia & $15(22)$ & 35 & 4 & 12 \\
\hline 23. Córdoba & $11(26)$ & 31 & 3 & 10 \\
\hline 24. Alicante & $27(17)$ & 30 & 7 & 10 \\
\hline 25. UNED & $22(18)$ & 30 & 5 & 10 \\
\hline 26. Castilla-La Mancha & $-\ldots$ & 22 & - & 7 \\
\hline 27. Málaga & $18(21)$ & 22 & 4 & 7 \\
\hline 28. Alcalá de Henares & $14(24)$ & 7 & 3 & 2 \\
\hline 29. León & $2(29)$ & 7 & 0,5 & 2 \\
\hline 30. Navarra & $12(25)$ & 6 & 3 & 2 \\
\hline 31. Deusto & $\ldots$ & 4 & - & 1 \\
\hline 32. Politécnica Canarias & $1(30)$ & 4 & 0,25 & 1 \\
\hline 33. Pontificia Comillas & $1(31)$ & 1 & 0,25 & 0,33 \\
\hline 34. Int. Menéndez Pelayo & $\ldots$ & 1 & $\ldots$ & 0,33 \\
\hline
\end{tabular}

* Entre paréntesis figura el número de orden para este período.

institución); así la toma de datos se ha hecho asignando las correspondientes fracciones a los trabajos realizados en colaboración (por mitades, cuando se trata de dos centros; por tercios si son tres).

El período de tiempo estudiado se corresponde con la fecha de publicación de los documentos originales. 


\section{Resultados}

El número total de documentos en la base de datos ICYT pertenecientes a las temáticas seleccionadas y correspondientes al periodo 1986-1988 es de 2.580; de ellos 1.436 corresponden a trabajos realizados en la Universidad lo que supone un $56 \%$, y el resto (1.144), a trabajos realizados en otros centros de investigación y empresas, $44 \%$.

Siguiendo la misma sistemática que en la parte 1, la figura 5 presenta los datos anuales de la producción en Física de la Universidad y de otros organismos. Se observa una tendencia general a la disminución del número de trabajos a lo largo del período, $-16 \%$.

Las tablas V y VI recogen las correspondientes distribuciones por tipos de centros y por Universidades, respectivamente. En la tabla V se observa una disminución en el número de documentos a lo largo del período, debida íntegramente al sector facultades, ya que los trabajos publicados por las ETS no han variado en número y los procedentes de los Colegios y Escuelas Universitarias han aumentado el $23 \%$.

Hay que destacar el elevado número de trabajos publicados por las facultades de Química y ello se debe a que en la clasificación de UNESCO la disciplina de Química-Física está contemplada dentro del campo de la Física y no en el de la Química. La distribución de trabajos por Organismos en esta disciplina corresponde en un $53 \%$ a la Facultad de Química, frente a un 5,5\% a la Facultad de Física.

El elevado número de documentos de la Facultad de Química en el año 86 se debe fundamentalmente a la publicación de una revista extra, editada con el conjunto de artículos presentados en una reunión científica celebrada en ese año. En cuanto al promedio de trabajos por centro figuran a la cabeza (excepción hecha de la ETSI Aeronáuticos, que es única) las ETSI de Telecomunicación e Industria-

Figura 5

Producción de la Universidad y otros organismos espanoles en ICYT

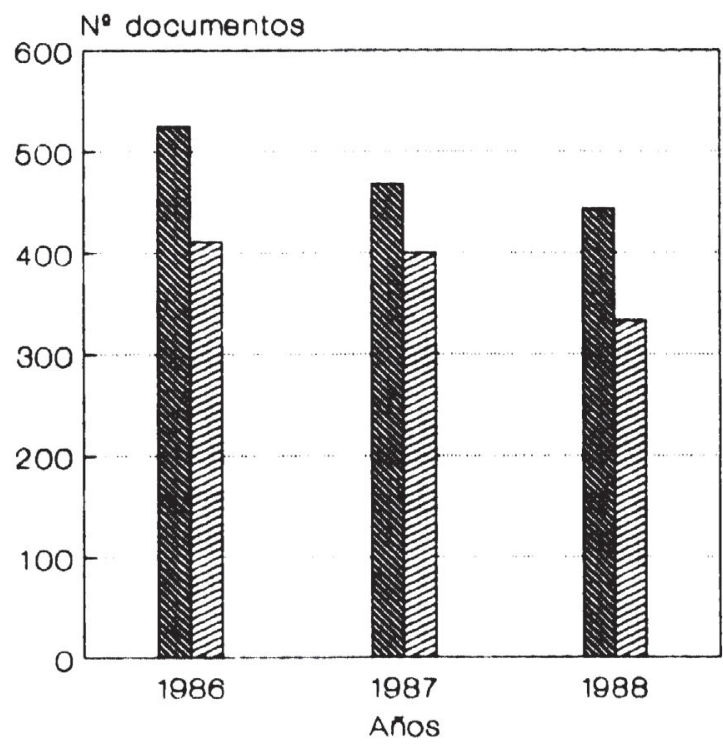

Universidad

Otros organismos 
Tabla V

Artículos de revistas por tipos de centro y promedios por centro (ICYT)

\begin{tabular}{lrrrrrr}
\hline & 1986 & 1987 & 1988 & Total & $\begin{array}{c}\text { Núm. de } \\
\text { centros }\end{array}$ & $\begin{array}{c}\text { Promedio/ } \\
\text { centro }\end{array}$ \\
\hline Fac. de Física & 90 & 112 & 75 & 277 & 21 & 13 \\
Fac. de Quimica & 145 & 100 & 75 & 320 & 24 & 13 \\
Fac. de Matemáticas & 5 & 12 & 4 & 21 & 8 & 3 \\
Fac. de Informática & 16 & 23 & 23 & 62 & 5 & 12 \\
Fac. de Geología & 5 & 9 & 7 & 21 & 8 & 3 \\
Fac. de Medicina & 7 & 5 & 6 & 18 & 7 & 3 \\
Fac. de Biología & 3 & - & - & 3 & 2 & 1 \\
Fac. de Farmacia & 26 & 13 & 13 & 52 & 7 & 7 \\
Fac. de Veterinaria & - & - & 2 & 2 & 2 & 1 \\
Otras Facultades & 2 & 2 & 3 & 7 & 6 & 1 \\
ETSI Industriales & 108 & 113 & 118 & 339 & 14 & 24 \\
ETSI Telecomunicación & 43 & 18 & 31 & 92 & 3 & 31 \\
ETS Arquitectura & 4 & 1 & - & 5 & 2 & 2 \\
ETSI Caminos & 6 & 5 & 3 & 14 & 4 & 3 \\
ETSI Minas & 9 & 9 & 12 & 30 & 2 & 15 \\
ETSI Aeronáuticos & 8 & 8 & 10 & 26 & 1 & 26 \\
ETSI Agrónomos & 4 & 1 & 5 & 10 & 3 & 3 \\
ETSI Montes & - & - & 2 & 2 & 1 & 2 \\
ETSI Navales & 1 & 1 & 1 & 3 & 1 & 3 \\
Insts. Universitarios & 6 & 2 & 2 & 10 & 8 & 1 \\
Esc. Univ. Técnicas & 29 & 25 & 39 & 93 & 31 & 3 \\
Colegios Universitarios & 7 & 6 & 10 & 23 & 6 & 4 \\
Esc. Univ. no Técnicas & 1 & 3 & 2 & 6 & 4 & 1 \\
\hline Total general & 525 & 468 & 443 & 1.436 & 170 & 8 \\
$\quad$ Total Facultades & & & & & & \\
(excl. Informática) & 283 & 253 & 185 & 721 & & \\
Total ETS & & & & & & \\
$\quad$ (incl. Informática) & 199 & 179 & 205 & 583 & & \\
\hline & & & & & & \\
\hline
\end{tabular}

les, seguidas a cierta distancia por las de Minas y las facultades de Química y de Física.

En la distribución por Universidades (tabla VI) destaca, como mayor productor, la Universidad Politécnica de Madrid, seguida de la Politécnica de Cataluña, la Universidad Complutense y la Universidad de Zaragoza con más de 100 documentos publicados durante el período estudiado. Un hecho a destacar en esta tabla es el incremento en el número de trabajos de la Universidad de Valencia en el año 88 en relación a los anteriores. 
Tabla VI

Distribución de los artículos de revista por Universidades y antos (ICYT)

\begin{tabular}{|c|c|c|c|c|}
\hline & 1986 & 1987 & 1988 & Total \\
\hline Politécnica de Madrid & 79 & 63 & 74 & 216 \\
\hline Politécnica de Cataluña & 54 & 40 & 51 & 145 \\
\hline Complutense & 43 & 56 & 33 & 132 \\
\hline Zaragoza & 41 & 25 & 35 & 101 \\
\hline Sevilla & 24 & 34 & 22 & 80 \\
\hline Politécnica de Valencia & 13 & 22 & 35 & 70 \\
\hline Barcelona & 32 & 25 & 12 & 69 \\
\hline Valladolid & 29 & 21 & 18 & 68 \\
\hline Valencia & 15 & 19 & 30 & 64 \\
\hline Granada & 26 & 21 & 12 & 59 \\
\hline País Vasco & 18 & 22 & 17 & 57 \\
\hline Santiago de Compostela & 10 & 15 & 9 & 34 \\
\hline Oviedo & 7 & 14 & 13 & 34 \\
\hline Autónoma Barcelona & 11 & 11 & 10 & 32 \\
\hline UNED & 9 & 8 & 12 & 29 \\
\hline Cantabria & 11 & 8 & 9 & 28 \\
\hline Salamanca & 18 & 3 & 7 & 28 \\
\hline Extremadura & 8 & 11 & 6 & 25 \\
\hline Autónoma Madrid & 5 & 13 & 6 & 24 \\
\hline Murcia & 12 & 6 & 6 & 24 \\
\hline Politécnica Canarias & 11 & 4 & 6 & 21 \\
\hline Córdoba & 14 & 4 & 1 & 19 \\
\hline Alicante & 9 & 5 & 2 & 16 \\
\hline La Laguna & 6 & 3 & 4 & 13 \\
\hline Málaga & 6 & 3 & 3 & 12 \\
\hline Navarra & 3 & 2 & 4 & 9 \\
\hline Alcalá de Henares & 2 & 3 & 3 & 8 \\
\hline Baleares & 5 & 2 & - & 7 \\
\hline Cádiz & 1 & 3 & 1 & 5 \\
\hline Pontificia Comillas & 1 & - & 2 & 3 \\
\hline Castilla-La Mancha & 1 & 1 & $\ldots$ & 2 \\
\hline León & 1 & - & - & 1 \\
\hline Deusto & - & 1 & $\cdots$ & 1 \\
\hline Total & 525 & 468 & 443 & 1.436 \\
\hline
\end{tabular}

\section{Comparación de los datos obtenidos en las bases de datos INSPEC e ICYT}

El objetivo principal de esta comparación consiste en analizar las tendencias de publicación en España y en el extranjero. Para ello, asumimos que las referencias de trabajos publicados en revistas españolas obtenidas de INSPEC están duplicadas en ICYT, pues esta base de datos vacía de forma exhaustiva los trabajos originales publicados en las revistas españolas. Tomamos, pues, como trabajos 
publicados en revistas españolas los obtenidos de la base de datos ICYT y como trabajos publicados en revistas extranjeras los obtenidos de INSPEC, una vez deducidos los que proceden de revistas españolas.

Téngase en cuenta, sin embargo, lo dicho anteriormente sobre el distinto modo en que se asignan los trabajos en cada base de datos, lo que hace que las cifras no sean rigurosamente comparables. Intervienen también otros factores, como distintos criterios de indización, etc. Ello desvirtúa la posibilidad de establecer relaciones entre la Universidad y otras instituciones. No obstante, como la diferencia derivada del distinto modo de asignación de los trabajos es pequeña y hay que suponer que afecte por igual a los distintos tipos de centros, entendemos que las comparaciones intra-universitarias sí son válidas.

La figura 6 presenta los datos globales de artículos publicados por la Universidad en revistas españolas y extranjeras. Se observa que, mientras el número de trabajos en revistas extranjeras crece en el trienio un $14 \%$, decrece casi en la misma proporción el número de trabajos publicados en revistas españolas $(-16 \%)$. Esto refleja una tendencia que se observa ya desde años anteriores a publicar preferentemente en revistas internacionales en lengua inglesa, en detrimento de las revistas españolas.

La tabla VII recoge la distribución por tipos de centros, en el mismo orden en que figuran en la tabla I para facilitar su comparación. En la última columna figura el aumento o disminución del porcentaje de publicaciones en el extranjero de 1986 a 1988. La tendencia a publicar en el extranjero es prácticamente el doble en las facultades que en las ETS (73 y $37 \%$, respectivamente) y esta diferencia crece a lo largo del período (incremento del $10 \%$ en las facultades y sólo del $5 \%$ èn las ETS). Parece que las escuelas técnicas tienden a realizar una investigación más aplicada, que se publica preferentemente en revistas locales, frente a una investigación más básica de las facultades, que presentan una mayor tendencia a la

Figura 6

Artículos publicados por la Universidad en revistas espanolas y extranjeras

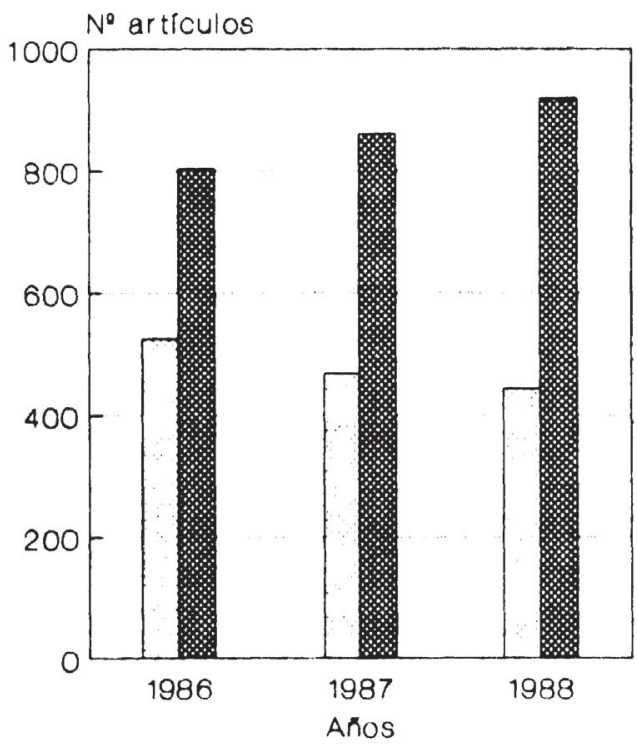

Rev.espenolas (ICYT)

Rev.extranj (INSPEC) 


\section{Tabla VII}

Artículos publicados en revistas espanolas (ICYT) y extranjeras (INSPEC) por tipo de centro (1986-88)

\begin{tabular}{lrrrrc}
\hline & Esp. & Extr. & Total & $\%$ Extr. & $\pm \%$ Extr. \\
\hline Fac. Físicas & 277 & 1.392 & 1.669 & 83 & +4 \\
Fac. Químicas & 320 & 516 & 836 & 62 & +17 \\
Fac. Matemáticas & 21 & 99 & 120 & 82 & +7 \\
Fac. Informática & 62 & 34 & 96 & 35 & -6 \\
Fac. Geología & 21 & 33 & 54 & 61 & -2 \\
Fac. Medicina & 18 & 21 & 39 & 54 & +4 \\
Fac. Biología & 3 & 20 & 23 & 87 & +25 \\
Fac. Farmacia & 52 & 11 & 63 & 17 & +12 \\
Fac. Veterinaria & 2 & 9 & 11 & 82 & -50 \\
Otras Facultades & 7 & 11 & 18 & 61 & +33 \\
ETSI Industriales & 339 & 142 & 481 & 29 & +7 \\
ETSI Telecomunicación & 92 & 91 & 183 & 50 & +9 \\
ETS Arquitectura & 5 & 24 & 29 & 83 & +33 \\
ETSI Caminos & 14 & 13 & 27 & 48 & +36 \\
ETSI Minas & 30 & 12 & 42 & 28 & +2 \\
ETSI Aeronáuticos & 26 & 18 & 44 & 41 & -6 \\
ETSI Agrónomos & 10 & 6 & 16 & 37 & +4 \\
ETSI Montes & 2 & - & 2 & - & - \\
ETSI Navales & 3 & - & 3 & - & - \\
Insts. Universitarios & 10 & 70 & 80 & 87 & +20 \\
Esc. Univ. Técnicas & 93 & 11 & 104 & 10 & -4 \\
Colegios Universitarios & 23 & 3 & 26 & 11 & +23 \\
Esc. Univ. no Técnicas & 6 & 4 & 10 & 40 & -50 \\
Sin especificar & - & 42 & 42 & 100 & - \\
\hline Total & & & & & \\
$\quad$ Total Facultades & 1.436 & 2.582 & 4.018 & 64 & +7 \\
(excl. Informática) & 721 & 2.112 & 2.833 & 74 & +10 \\
Total ETS & & & & & \\
$\quad$ incl. Informática) & 583 & 340 & 923 & 37 & +5 \\
\hline & & & & & \\
\hline
\end{tabular}

publicación en el extranjero. Si tomamos los cuatro tipos de centros más representativos, la tendencia es mayor en los típicamente «fisicos» (Fac. de Físicas y Esc. de Telecomunicación) que en los "afines" (Fac. de Químicas y Esc. de Industriales). Pero el incremento a lo largo del período es máximo en las facultades de Química $(17 \%)$ y mínimo en las de Física $(4 \%)$.

La tabla VIII presenta la distribución por Universidades, en orden decreciente del número total de trabajos. El mayor porcentaje de publicaciones extranjeras lo presenta la Universidad Autónoma de Madrid, con el $92 \%$ de sus publicaciones en revistas internacionales. Si limitamos el análisis a las 16 Universidades cuya aportación es superior a 100 artículos (y que en conjunto cubren el $84 \%$ del total 


\section{Tabla VIII}

Artículos publicados en revistas espanolas (ICYT) y extranjeras (INSPEC) por Universidades (1986-88)

\begin{tabular}{|c|c|c|c|c|}
\hline & Esp. & Extr. & Total & $\%$ Extr. \\
\hline Complutense & 132 & 278 & 410 & 68 \\
\hline Politécnica Madrid & 216 & 127 & 343 & 37 \\
\hline Barcelona & 69 & 266 & 335 & 79 \\
\hline Autónoma Madrid & 24 & 284 & 308 & 92 \\
\hline Politécnica Cataluña & 145 & 123 & 268 & 46 \\
\hline Zaragoza & 101 & 150 & 251 & 60 \\
\hline Sevilla & 80 & 129 & 209 & 62 \\
\hline Valencia & 64 & 139 & 203 & 68 \\
\hline País Vasco & 57 & 140 & 197 & 71 \\
\hline Valladolid & 68 & 100 & 168 & 59 \\
\hline Autónoma de Barcelona & 32 & 131 & 163 & 80 \\
\hline Granada & 59 & 65 & 124 & 52 \\
\hline Cantabria & 28 & 79 & 107 & 74 \\
\hline Politécnica Valencia & 70 & 36 & 106 & 34 \\
\hline Santiago de Compostela & 34 & 70 & 104 & 67 \\
\hline Oviedo & 34 & 68 & 102 & 67 \\
\hline La Laguna & 13 & 82 & 95 & 86 \\
\hline Salamanca & 28 & 54 & 82 & 66 \\
\hline Extremadura & 25 & 35 & 60 & 58 \\
\hline UNED & 29 & 27 & 56 & 48 \\
\hline Murcia & 24 & 28 & 52 & 54 \\
\hline Córdoba & 19 & 27 & 46 & 59 \\
\hline Cádiz & 5 & 40 & 45 & 89 \\
\hline Baleares & 7 & 37 & 44 & 84 \\
\hline Alicante & 16 & 27 & 43 & 63 \\
\hline Málaga & 12 & 20 & 32 & 62 \\
\hline Politécnica Canarias & 21 & 1 & 22 & 4 \\
\hline Alcalá de Henares & 8 & 6 & 14 & 43 \\
\hline Navarra & 9 & 4 & 13 & 31 \\
\hline León & 1 & 6 & 7 & 86 \\
\hline Castilla-La Mancha & 2 & 2 & 4 & 50 \\
\hline Pontificia de Comillas & 3 & 1 & 4 & 50 \\
\hline Deusto & 1 & - & 1 & $-\cdots$ \\
\hline Total & 1.436 & 2.582 & 4.018 & 64 \\
\hline
\end{tabular}

de éstos), veremos que, en lo que se refiere a la tendencia a publicar en el extranjero, se pueden dividir en tres grupos:

a) Las que tienen un porcentaje de publicación en el extranjero superior al $75 \%$ : Universidades Autónomas de Madrid y Barcelona y Universidad de Barcelona. 
b) Aquéllas en que dicho porcentaje está entre el 50 y el $75 \%$; es el grupo más numeroso: Cantabria, País Vasco, Complutense, Valencia, Santiago, Oviedo, Sevilla, Zaragoza, Valladolid y Granada.

c) Las que presentan un porcentaje de publicación en el extranjero inferior al $50 \%$ : Politécnica de Cataluña, Politécnica de Madrid y Politécnica de Valencia.

Se llega pues, por una parte, a la misma conclusión citada anteriormente, en cuanto al diferente comportamiento de facultades y escuelas técnicas y, por otra parte, destaca la tendencia a publicar en el extranjero en las Universidades Autónomas de Madrid y Barcelona (especialmente en aquélla), fenómeno que ya habíamos observado en 1971-77 (1) y que ha seguido acentuándose en los años siguientes.

\section{Conclusiones}

1. La participación de la Universidad en la producción científica española en el área de la Física es muy importante. Los trabajos en que ha participado la Universidad representan el $83 \%$ del total de la aportación española en la base de datos INSPEC y el $56 \%$ en la base de datos ICYT.

2. Esta producción se publica mayoritariamente en revistas extranjeras $(64 \%$, deducido de la comparación entre ambas bases de datos). El porcentaje de publicación en el extranjero sube, a lo largo del trienio, del $60 \%$ en 1986 al $67 \%$ en 1988. Esta tendencia a publicar más en revistas extranjeras y menos en españolas refleja una actitud general de los científicos, pues, por una parte, consiguen una mayor difusión internacional de sus artículos, y además una mejor consideración de cara a "curriculum» (oposiciones, sexenios, etc.).

3. Por tipos de centros, ocupan los primeros lugares las facultades de Física y Química y las ETSI de Telecomunicación e Industriales, tanto en números absolutos como en promedio de trabajos por centro y en las dos bases de datos (en promedios por centro aparece también la ETSI Aeronáuticos, si bien se trata de un único centro).

4. El conjunto de facultades (artículos de revista) representa un $79 \%$ del total en INSPEC y un $50 \%$ en ICYT, mientras que el conjunto de ETS (incluidas las facultades de Informática) representa respectivamente el 17 y el $40 \%$ en cada base de datos. La tendencia a publicar en el extranjero es prácticamente el doble en las facultades ( 73 frente a $37 \%$ ). Entre las causas de mayor publicación de ETS en revistas españolas figura el hecho de que su investigación es de tipo más aplicado que la que desarrollan las facultades y, por ende, de un ámbito de interés más local.

5. Por Universidades, la situación es bastante diferente en las dos bases de datos, como cabría esperar a la vista de la distinta tendencia a publicar en el extranjero de facultades y ETS. En INSPEC los primeros lugares están ocupados por las Universidades Complutense y Autónoma de Madrid y por la Universidad de Barcelona, mientras en ICYT son las dos Universidades 
Politécnicas de Madrid y Cataluña las que ocupan los primeros puestos, seguidas por la Complutense. En cuanto a publicaciones en el extranjero, destacan los porcentajes de las Universidades Autónomas de Madrid y Barcelona, mientras que las Universidades Politécnicas presentan, en general, un porcentaje de publicación en el extranjero relativamente bajo.

6. En comparación con el período 1982-85 (efectuada únicamente en INSPEC), el promedio anual de trabajos ha aumentado el $62 \%$. Pero mientras las facultades han aumentado el $44 \%$, las ETS lo han hecho en el $105 \%$.

\section{Bibliografia}

1. VIESCA, R. ; PEREZ AlVAREZ-OSSORIO, J. R. Análisis de la literatura española en Física a través del banco de datos del INSPEC (sección Física). Rev. Esp. Doc. Cient., 1 (1), 57-63, 1977.

2. LOPEZ AGUADO, G.; VIESCA, R. Trabajos científicos publicados por los físicos españoles y recogidos en INSPEC durante 1978-79. Rev. Esp. Doc. Cient., 6 (4), 317-330, 1983.

3. GOMEZ CARIDAD, I.; MENDEZ, A. La producción científica de la Universidad Española recopilada por las bases de datos internacionales en 1985. Madrid, 1986.

4. FERNANDEZ CALEYA, R. ; PASCUAL, P. Algunos datos sobre la evolución de la física en España. Prospectiva en Fisica, ANEP, CSIC, 1988.

5. FERREIRO ALAEZ, L.; LOPEZ AGUADO, G.; VIESCA, R. International integration of Spanish authors in Physics, Czechoslovak Journal of Physics, B 36, 47-51, 1986. 
En esta sección se pretende incluir los proyectos de normas españolas relativas a información y documentación, durante el periodo en que éstas están sometidas a preceptiva información pública.

En este número se recoge el siguiente proyecto de Norma Española (PNE) aprobado por el Comité Técnico de Normalización núm. 50 de AENOR, Documentación.

Cualquier observación respecto a su contenido deberá dirigirse a AENOR, Fernández de la Hoz, 52, 28010 Madrid.

La versión definitiva del proyecto de norma que se recoge a continuación variará en función de las observaciones formuladas.

\section{DOCUMENTACION - PAGINAS DE RESUMENES EN LAS PUBLICACIONES PERIODICAS ISO 5122-1979 UNE-50-112}

\section{Introducción}

La página de resúmenes en una publicación periódica o en serie proporciona una descripción detallada de cada artículo, aportando aquellos elementos que son esenciales para el trabajo documental. La página se divide en bloques; cada uno contiene información sobre un articulo, ordenados de tal forma que los detalles de menos interés, que están en la cabecera y en el pie del bloque, se pueden suprimir cuando se consideren superfluos. La cabecera de la página de resúmenes suministra la información suplementaria necesaria para obtener el documento original. La página de resúmenes no puede de ninguna forma sustituir al sumario de la publicación.

Cuando los editores prefieren que un resumen acompañe a cada artículo, se recomienda que la presentación sea la misma que para la página de resúmenes.

\section{Objeto y campo de aplicación}

Esta Norma establece directrices para la presentación de una página de resúmenes en publicaciones periódicas o en serie.

\section{Normas para consulta}

UNE-1-008.-Documentación.-Código Internacional para la abreviatura de los títulos de publicaciones periódicas.

UNE-50-101.-Documentación.--Presentación de las publicaciones periódicas.

UNE-50-103.-Documentación.-Preparación de resúmenes.

UNE-1-071.-- Presentación de los artículos de publicaciones periódicas.

UNE-1-091.- Símbolos para idiomas, paises y autoridades. 